\title{
The anaphase-promoting complex/cyclosome (APC/C) is required for rereplication control in endoreplication cycles
}

\author{
Norman Zielke, ${ }^{1,3}$ Silvia Querings, ${ }^{1}$ Carmen Rottig, ${ }^{2}$ Christian Lehner, ${ }^{2}$ and Frank Sprenger ${ }^{1,2,4}$ \\ ${ }^{1}$ University of Cologne, Institute for Genetics, 50674 Köln, Germany; ${ }^{2}$ University of Zürich, Institute of Zoology, \\ CH-8057 Zürich, Switzerland
}

Endoreplicating cells undergo multiple rounds of DNA replication leading to polyploidy or polyteny. Oscillation of Cyclin E (CycE)-dependent kinase activity is the main driving force in Drosophila endocycles. High levels of CycE-Cdk2 activity trigger S phase, while down-regulation of CycE-Cdk2 activity is crucial to allow licensing of replication origins. In mitotic cells relicensing in $S$ phase is prevented by Geminin. Here we show that Geminin protein oscillates in endoreplicating salivary glands of Drosophila. Geminin levels are high in S phase, but drop once DNA replication has been completed. DNA licensing is coupled to mitosis through the action of the anaphase-promoting complex/cyclosome (APC/C). We demonstrate that, even though endoreplicating cells never enter mitosis, APC/C activity is required in endoreplicating cells to mediate Geminin oscillation. Down-regulation of APC/C activity results in stabilization of Geminin protein and blocks endocycle progression. Geminin is only abundant in cells with high CycE-Cdk2 activity, suggesting that APC/C-Fzr activity is periodically inhibited by CycE-Cdk2, to prevent relicensing in S-phase cells.

[Keywords: Anaphase-promoting complex; cell cycle; DNA licensing; Drosophila; endoreplication]

Supplemental material is available at http://www.genesdev.org.

Received December 21, 2007; revised version accepted April 8, 2008.

The precise duplication of the genome is crucial for the survival of any organism. In multicellular organisms genome instability potentially gives rise to cancer and thus compromises the life of the whole organism. To maintain the integrity of the genome, DNA replication, and mitosis must be coordinated during cell division cycles so that DNA replication occurs only once per cycle and mitosis only after complete duplication of the genome. To avoid rereplication events, a network of proteins ensures that cells acquire the license for DNA replication only in a specific phase of the cell cycle. DNA licensing involves the formation of prereplication complexes (pre$\mathrm{RC})$, which can only assemble during late mitosis and G1 (for review, see Bell and Dutta 2002; Blow and Dutta 2005). Pre-RC assembly on replication origins involves a variety of conserved components including the ORC1-6 complex, Cdc6, Cdt1/Double-parked, and the MCM2-7 helicase. Once MCM2-7 proteins are loaded onto the

\footnotetext{
${ }^{3}$ Present address: Fred Hutchinson Cancer Research Center, 1100 Fairview Ave. North, P.O. Box 19024, Seattle, Washington 98109, USA. ${ }^{4}$ Corresponding author.

E-MAIL frank.sprenger@zool.uzh.ch; FAX 41-0-44-6356820.

Article is online at http://www.genesdev.org/cgi/doi/10.1101/gad.469108.
}

DNA, licensing is completed and the replication origins are ready for firing (for review, see Bell and Dutta 2002; Blow and Dutta 2005).

Several mechanisms ensure that replication origins are fired only once during a cell cycle (for review, see Machida et al. 2005). Of particular importance is the temporal separation of pre-RC formation and origin firing. Pre-RC assembly occurs only in G1 because it depends on low Cdk activity while high Cdk activity is required for origin firing. The increase in Cdk activity at the G1-S transition does not only trigger origin firing, but at the same time also results in phosphorylation of several preRC components (ORC, Cdc6, Cdt1, and MCM2-7). These modifications are thought to prevent reassembly of these components into pre-RCs. Moreover, ongoing DNA replication results in Cdt1 instability mediated by a Cul4-dependent pathway (for review, see Arias and Walter 2007). In addition, pre-RC formation in S phase is blocked by Geminin, a protein that binds to Cdt1 and thereby inhibits loading of MCM2-7 proteins onto the chromatin (Wohlschlegel et al. 2000; Tada et al. 2001). Geminin accumulates during S, G2, and M phase but is destabilized during G1 by the action of an ubiquitin ligase, the anaphase-promoting complex/cyclosome 
(APC/C) (McGarry and Kirschner 1998). The APC/C has originally been identified because of its important role during mitosis (for review, see Peters 2006; Pines 2006). However, APC/C activity is also observed during G1 where it is crucial for the control of pre-RC formation, because it mediates the proteasomal degradation of Geminin and A/B-type cyclins. The APC/C-mediated proteasomal degradation of Geminin results in the release of Cdt1, and cyclin degradation creates a window of low Cdk1 activity, thereby allowing pre-RC formation. Recently this has been corroborated by analyzing cells in which Emil, a negative regulator of APC/C activity, has been depleted. In these cells the APC/C is continuously activated, causing degradation of Geminin and A/B-type cyclins, which results in uncoordinated overreplication of the DNA (Di Fiore and Pines 2007; Machida and Dutta 2007). Analysis of imaginal disc cells depleted of Rcal, the Drosophila ortholog of Emil (Grosskortenhaus and Sprenger 2002), revealed that this mechanism of rereplication control also applies to flies (Supplemental Material).

While rereplication without an intervening mitosis is strictly prevented during cell division cycles, this process is enforced during endoreplication cycles. This cell cycle variant is prominently found during plant and invertebrate development, but some mammalian cell types, like megakaryocytes or trophoblast giant cells, are also known to undergo endoreplication cycles (for review, see Edgar and Orr-Weaver 2001; Lilly and Duronio 2005). Endoreplicating cells undergo repeated rounds of DNA synthesis that are separated by distinct Gap phases (from here referred to as $\mathrm{G}$ phase), but never enter mitosis. In the last decade several studies have demonstrated that the proteins required for entry and progression through $S$ phase during mitotic cycles are also essential for DNA replication during endoreplication cycles (for review, see Edgar and Orr-Weaver 2001; Lilly and Duronio 2005). However, it is still poorly understood how DNA licensing is regulated during endoreplication cycles. Drosophila Cyclin E (CycE) and its designated kinase partner Cdk2 are required for $\mathrm{S}$ phase during both mitotic and endoreplication cycles (Knoblich et al. 1994; Lane et al. 2000). Several studies have demonstrated that CycE levels oscillate in endoreplicating tissues (Lilly and Spradling 1996; de Nooij et al. 2000; Weng et al. 2003). The periodicity of CycE-Cdk2 activity appears to be essential for endoreplication, since continuous CycE expression prevents DNA replication in endocycling cells (Follette et al. 1998; Weiss et al. 1998), although the affected downstream targets have never been identified.

Another regulator that has been implicated in the control of endoreplication is Fizzy-related (Fzr), the Drosophila ortholog of the APC/C activator Cdh1. Analysis of $f z r$ mutants revealed that APC/C-Fzr activity is crucial for the transition from mitotic to endoreplication cycles (Sigrist and Lehner 1997; Schaeffer et al. 2004). At this step, APC/C activity is required to down-regulate A/B-type cyclins to prevent another round of mitotic proliferation. However, since expression of the main Fzr targets, the A/B-type cyclins, has not been reported in endoreplicating tissues, it has been suggested that APC/ C-Fzr activity might only be required for the transition from mitotic to endoreplication cycles (Edgar and OrrWeaver 2001; Lilly and Duronio 2005). The requirement of Fzr during endoreplication cycles has never been tested experimentally. Furthermore, it has not been addressed whether Geminin is involved in endoreplication. As Geminin-independent rereplication control is entirely sufficient during mitotic cycles in budding yeast, which does not have a Geminin ortholog (McGarry and Kirschner 1998), it was widely assumed that rereplication control during Drosophila endoreplication cycles relies on a mechanism resembling those in yeast (for review, see Bell and Dutta 2002). However, here we show that the APC/C-Fzr complex mediates the fluctuation of Geminin protein in endoreplicating salivary glands and demonstrate that APC/C-Fzr activity is required for normal endoreplication.

\section{Results}

rcal is not required for rereplication control in endoreplicating cells

In Drosophila, a variety of tissues is comprised of polyploid or polytene cells. These cells undergo endoreplication cycles, a cell cycle variant that is characterized by periodic DNA replication and the lack of $M$ phases. The involvement of the APC/C inhibitor proteins, Emil and Rcal, in rereplication control during mitotic cycles (Supplemental Material; Di Fiore and Pines 2007; Machida and Dutta 2007) raised the question whether Rcal activity is also required during endocycles. Therefore, we generated rca1 mutant cell clones in salivary glands that endoreplicate extensively during the larval stages. The rca1 mutant clones were induced by mitotic recombination using the MARCM technique (Lee and Luo 1999). Since mitotic recombination only occurs in proliferating cells, clones were already induced during embryogenesis when the salivary gland precursor cells still divide mitotically. Inspection of DNA contents at the third instar stage revealed that the DNA staining in rcal mutant cells (marked by GFP) is indistinguishable from those of the surrounding control cells. This indicates that rca1 is not essential for progression through endoreplication cycles.

Expression of mitotic genes and Fzr in endoreplicating cells

Our finding that Rcal is not required during endocycles suggested that the APC/C, whose activity needs to be restricted by Rcal during mitotic cycles, might not have a role during endoreplication cycles either. After initiation of the first G1 phase during embryogenesis, virtually all cells of internal organs undergo endoreplication (Smith and Orr-Weaver 1991). Previous studies have clearly established that APC/C-Fzr activity is required 
for the transition form mitotic to endocycles during embryogenesis (Sigrist and Lehner 1997), but a later requirement of APC/C activity during progression through endoreplication cycles has not yet been addressed. Moreover, because a variety of proteins targeted for proteasomal degradation by the APC/C during mitotic cycles are transcriptionally down-regulated in endoreplicating cells (Klebes et al. 2002), the APC/C has often been assumed to be dispensable during endoreplication cycles. To determine the role of APC/C during endoreplication cycles, we started a careful comparison of transcript levels of selected genes encoding APC/C subunits including Fzr, as well as some cell cycle regulators during mitotic and endoreplication cycles. RNA derived from embryos (4-8 h after egg deposition [AED]) where cells proliferate mitotically and endoreplicating salivary glands from feeding larvae were analyzed by quantitative RT-PCR (qRT-PCR). This approach revealed that transcript levels of the tested cell cycle genes were generally reduced in salivary glands, but the degree of reduction varied considerably (Fig. 1B). Levels of genes required for $S$ phase (E2F1, CycE, Cdk2, dup/Cdt1, and Rnr2) were moderately decreased in salivary glands compared with embryos (10- to 100-fold). Most genes involved in Mphase control (rca1, Cdk1, string/Cdc25, Cyclin A, and Cyclin B) displayed a far stronger reduction (400- to 8000-fold). Importantly, Fzr as well as the two APC/C subunits morula/APC2 (mr) and Cdc16/APC6 (Reed and Orr-Weaver 1997; Kashevsky et al. 2002; Pal et al. 2007) were expressed at levels similar to those of the S-phase regulators, which are known to be involved in endoreplication. We also compared protein levels of the APC/C activator Fzr in embryonic protein extracts and salivary glands (Fig. 1C). Consistent with its embryonic function, Fzr was readily detectable in extracts derived from 7 - to 9-h-old embryos. In larval salivary glands, Fzr levels were only slightly reduced. Therefore, we conclude that Fzr is expressed at significant levels in endoreplicating salivary glands even though the A/B-type cyclins, which are known APC/C-Fzr targets, are transcriptionally downregulated.

\section{Overexpression of HA-Rca1 impairs endocycle progression}

The finding that APC/C-Fzr is present at significant levels in larval salivary glands suggested that APC/C activity might be required during endocycles. To address this possibility we ectopically expressed the APC/C-Fzr inhibitor Rcal (Grosskortenhaus and Sprenger 2002) at different time points after the initiation of endoreplication. For conditional expression of UAS-HA-Rcal we used ptc-Gal4, which is active in salivary glands (Pierce et al. 2004), in combination with a temperature-sensitive variant of the Gal4 inhibitor Gal80 (McGuire et al. 2004). When larvae were cultured at $18^{\circ} \mathrm{C}$ (permissive temperature for Gal80 ${ }^{\text {ts }}$ ), salivary glands did not display HA-Rca 1 staining (Fig. 2A) and reached the same size as controls. In contrast, salivary glands derived from larvae cultured at $29^{\circ} \mathrm{C}$ (restrictive temperature for $\mathrm{Gal}^{\circ} 0^{\text {ts }}$ ), were posi- tive for HA-Rcal and dramatically smaller than control glands. The nuclei in these salivary glands were accordingly reduced in size and even smaller than the surrounding fat body cells that do not express HA-Rcal (Fig. 2B); demonstrating that excess HA-Rcal inhibits endoreplication effectively. Early overexpression of HA-Rcal is expected to interfere with the established role of APC/C-Fzr activity at the switch from mitotic to endoreplication cycles (Sigrist and Lehner 1997). However, HA-Rcal overexpression also inhibited salivary gland endoreplication when it was induced between 10 and $18 \mathrm{~h} \mathrm{AED,} \mathrm{when} \mathrm{cells} \mathrm{of} \mathrm{the} \mathrm{salivary} \mathrm{gland} \mathrm{had} \mathrm{al-}$ ready entered the endoreplication program. The resulting salivary glands were significantly smaller in size, albeit slightly bigger than those that constantly overexpressed HA-Rcal (Fig. 2C). Even induction at 40-48 h AED resulted in significantly smaller salivary glands when compared with wild type (Fig. 2D). Finally, we generated mosaic salivary glands using the flp-out technique (Ito et al. 1997) to analyze the effect of Rcal overexpression in individual cells. When Rcal expression was induced at 40-48 h AED, we found that cells expressing high levels of HA-Rcal contained less DNA compared with the adjacent wild-type cells (Fig. 2E).

\section{Depletion of APC/C activity compromises endoreplication}

To corroborate our finding that HA-Rcal overexpression, impairs endocycle progression in larval salivary glands we performed BrdU labeling. Larvae were fed with BrdU for $24 \mathrm{~h}$ and antibody staining was used for visualization of incorporated BrdU in salivary glands. All nuclei of wild-type salivary glands showed BrdU staining (Fig. 3A). However, after HA-Rcal overexpression, induced at 10$18 \mathrm{~h}$ AED, no BrdU incorporation was observed within salivary glands, although it was readily detectable in the adjacent fat body (Fig. 3B). A very similar phenotype was observed after overexpression of CycE in endoreplication salivary glands (Fig. 3C; Follette et al. 1998; Weiss et al. 1998). Continuous CycE expression is known to interfere with DNA replication in salivary glands, and thus we conclude that progression through endocycle $S$ phases is inhibited by HA-Rcal overexpression.

To verify that suppression of APC/C activity impairs endoreplication, we depleted $C d c 16$, a core component of the APC/C (Huang and Raff 2002; Pal et al. 2007). Overexpression of an RNAi construct targeting Cdc16 in imaginal disc cells resulted in a mitotic arrest phenotype (Supplemental Fig. S2), as expected from a knockdown of APC/C function (Deak et al. 2003; Pal et al. 2007). Expression of the Cdc16-specific UAS-RNAi construct in salivary glands, using ptc-Gal4 in combination with Gal80 ${ }^{\text {ts }}$, was induced at $10-18 \mathrm{~h}$ AED to deplete APC/C function after the onset of endoreplication. The resulting salivary glands displayed a reduced size (Fig. 4D; Supplemental Fig. S3) and a strong reduction in BrdU incorporation when assayed in the third instar stage (Fig. 4D). In many cells, we observed unusual BrdU incorporation into small dots that were never seen in wild type (Fig. 

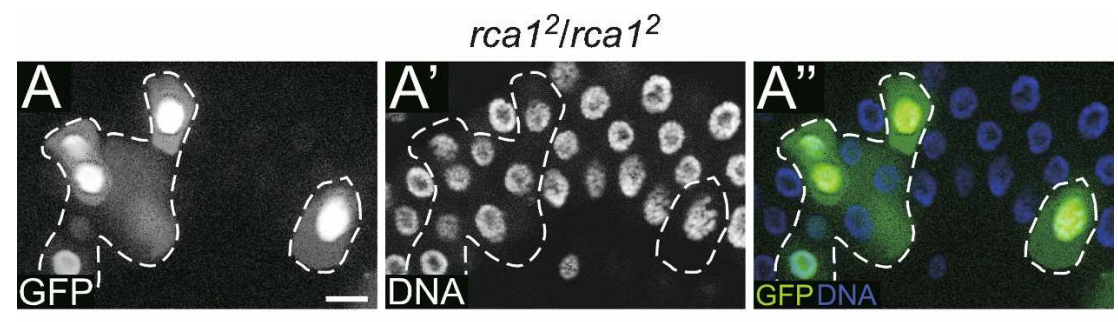

B

log10(relative quantity)
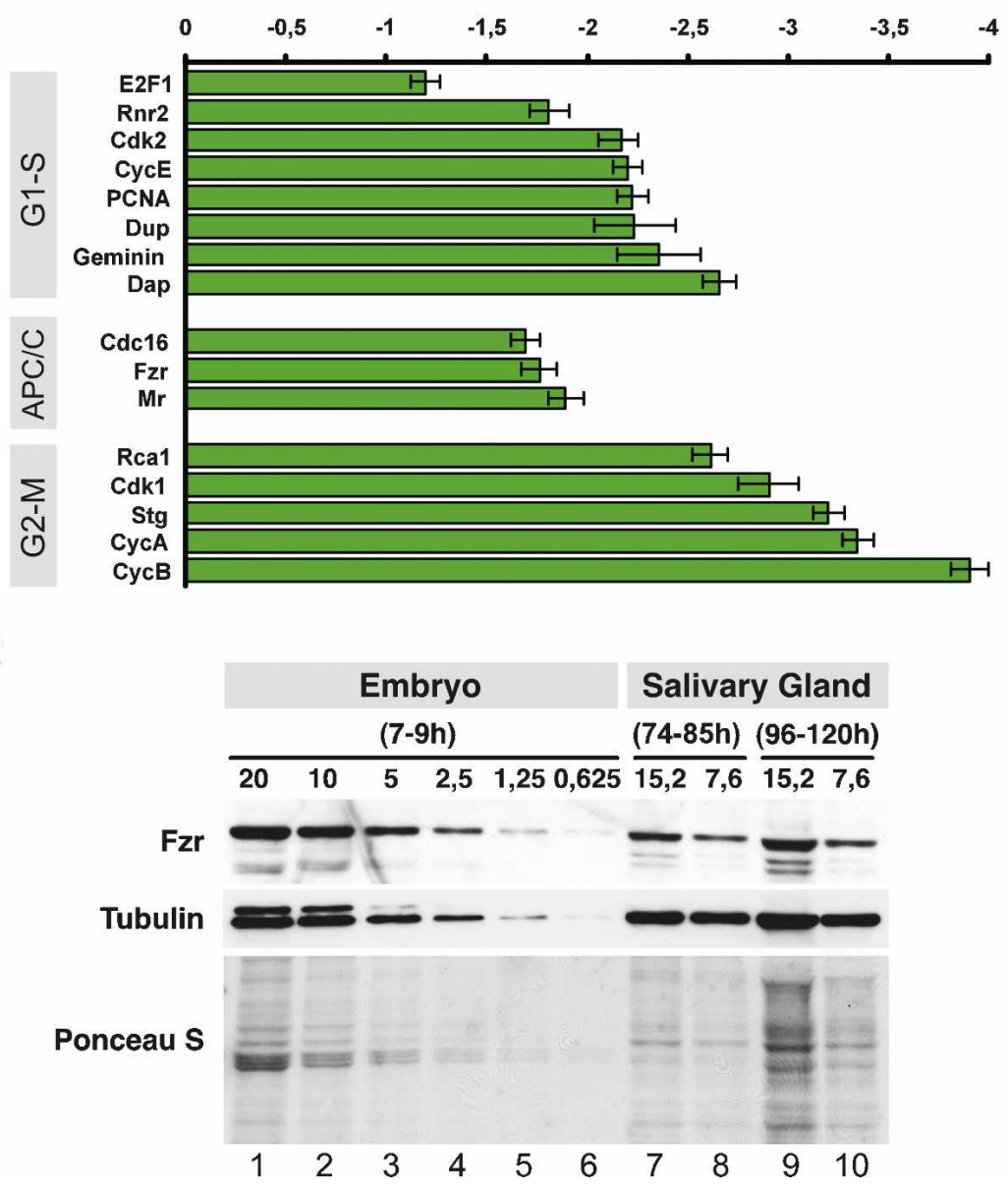

Figure 1. Expression of APC/C components in salivary glands. (A) Endocycle progression is not affected in $\mathrm{rca}^{2}$ mutant cells. Section of a third instar salivary gland containing cells mutant for $\mathrm{rca}^{2}$ that were generated in the embryonic salivary placode by the MARCM technique and were positively marked by GFP. DNA staining reveals no difference in nuclear size between $\mathrm{rca}^{2}$ mutant and adjacent control cells, indicating that Rcal is not essential for endocycle progression in salivary glands. Bar, $50 \mu \mathrm{m}$. (B) qRTPCR analysis of transcript levels in salivary glands. The relative expression of the indicated genes in feeding third instar salivary glands and embryos (4-8 h AED) was determined by the $\Delta \Delta \mathrm{CT}$ method. GAPDH was used as endogenous control to normalize expression between both cDNA preparations. In salivary glands all tested transcripts were expressed at lower levels when compared with embryos. However, expression levels of mitotic genes $(\mathrm{G} 2-\mathrm{M})$ were dramatically reduced compared with genes involved in G1-S control (e.g., CycE, Cdk2), which are known to be essential for endoreplication. APC/C components, including the activator protein Fzr, were expressed at levels comparable with those of the G1-S genes. (C) Fzr protein is present in larval salivary glands. Extracts from embryos and salivary glands of indicated time after egg deposition were analyzed by Western blotting. The blot was probed with antibodies against Fzr and Tubulin. Ponceau S staining is shown as a reference of total protein levels. Several dilutions were tested to compare signal intensities. The number of embryos or salivary glands per lane is indicated. Lanes 2, 7, and 10 show roughly similar signal intensities of tubulin and Ponceau S. In these lanes, similar levels of Fzr were detectable, indicating that embryos and larval salivary glands contain similar concentrations of Fzr protein.
4D). A few cells showed almost normal BrdU incorporation indicating that the RNAi-mediated knockdown of Cdc16 is variable. Altogether, inhibition of APC/C-Fzr activity either by depletion of $C d c 16$ or Rcal overexpression impairs BrdU incorporation in endoreplicating salivary glands. These experiments demonstrate that APC/C activity is not only required for the switch from mitotic to endoreplication cycles but that it is also crucial for the maintenance of endoreplication.

\section{APC/C-depleted cells exhibit hallmarks of increased CycE-Cdk2 activity}

The similarity between the Rcal and CycE overexpression phenotypes prompted us to test whether inactivation of the APC/C-Fzr complex results in persistently high CycE-Cdk2 activity. In Drosophila, the monoclonal MPM-2 antibody is widely used to monitor CycECdk2 activity (Calvi et al. 1998; Royzman et al. 1999). The MPM-2 antibody recognizes a Cdk2-regulated protein that assembles into the histone locus body (White et al. 2007). The presence of MPM-2-positive nuclear spheres correlates with CycE-Cdk2 activity. In wildtype salivary glands dissected from wandering third instar larvae that had finished endoreplication and were thus supposed to have low CycE-Cdk2 activity, we did not detect these MPM-2-positive nuclear spheres, as expected (Fig. 4A). By contrast, salivary glands overexpressing CycE showed MPM-2-positive spheres in virtually all nuclei (Fig. 4B). Overexpression of HA-Rcal also resulted in the appearance of MPM-2 epitopes (Fig. 4C). In addition to MPM-2-positive nuclear spheres, both CycE and 
Figure 2. Expression of Rcal impairs endoreplication in larval salivary glands. Spatially and temporally expression of HARcal was achieved by ptc-Gal4 and the TARGET system that is based on a temperature-sensitive form of the Gal4 inhibitor Gal80 (Gal80 $\left.{ }^{\text {ts }}\right)$. Ptc-Gal4 activates expression specifically in the salivary gland $(\mathrm{sg})$ but not in the neighboring fat body $(\mathrm{fb})$. (A) At restrictive temperature for $\mathrm{Gal} 80^{\text {ts }}\left(18^{\circ} \mathrm{C}\right)$, salivary development occurred normally and neither HA-Rcal nor the coexpressed GFP was detectable. (B) At $29^{\circ} \mathrm{C}$, Gal $80^{\text {ts }}$ is inactive, allowing Gal4 to activate UAS-HA-Rcal (and UAS-GFP) resulting in very small salivary glands compared with controls. $(C)$ When expression was triggered $10-18 \mathrm{~h}$ AED by a shift from permissive to restrictive temperature, salivary glands with reduced size were still observed. $(D)$ Even when the temperature shift was performed at 40-48 h AED salivary gland development was severely impaired. When expression was induced at the two latter time points, cells had already passed the transition from mitotic to endoreplication cycles, thus demonstrating that HA-Rcal overexpression perturbs endocycle progression and not initiation. Bar, $100 \mu \mathrm{m}$. (E) Overexpression of HARcal in individual salivary gland cells using the flp-out technique. Expression was induced at 40-48 h AED. HA-Rca1expressing cells were marked by coexpression of GFP and HA staining. Overexpression of HA-Rcal results in smaller cells with reduced DNA content compared with the neighboring control cells. Bar, $25 \mu \mathrm{m}$.

HA-Rcal expression resulted in a conspicuous pattern of DNA staining with a bright speckle in each nucleus, which likely corresponds to heterochromatin that is known to bind the DNA-staining dye more efficiently. Importantly, these phenotypic abnormalities (MPMpositive nuclear spheres and heterochromatin speckles) were also induced by RNAi-mediated depletion of Cdc16 (Fig. 4D). In summary, these experiments indicate that $\mathrm{APC} / \mathrm{C}$ inactivation in endoreplicating salivary glands stimulates CycE/Cdk1 activity.

\section{APC/C inactivation increases CycE level through E2F1 stabilization}

CycE-Cdk2 activity appears to be elevated in APC/Cdepleted salivary glands, raising the question whether
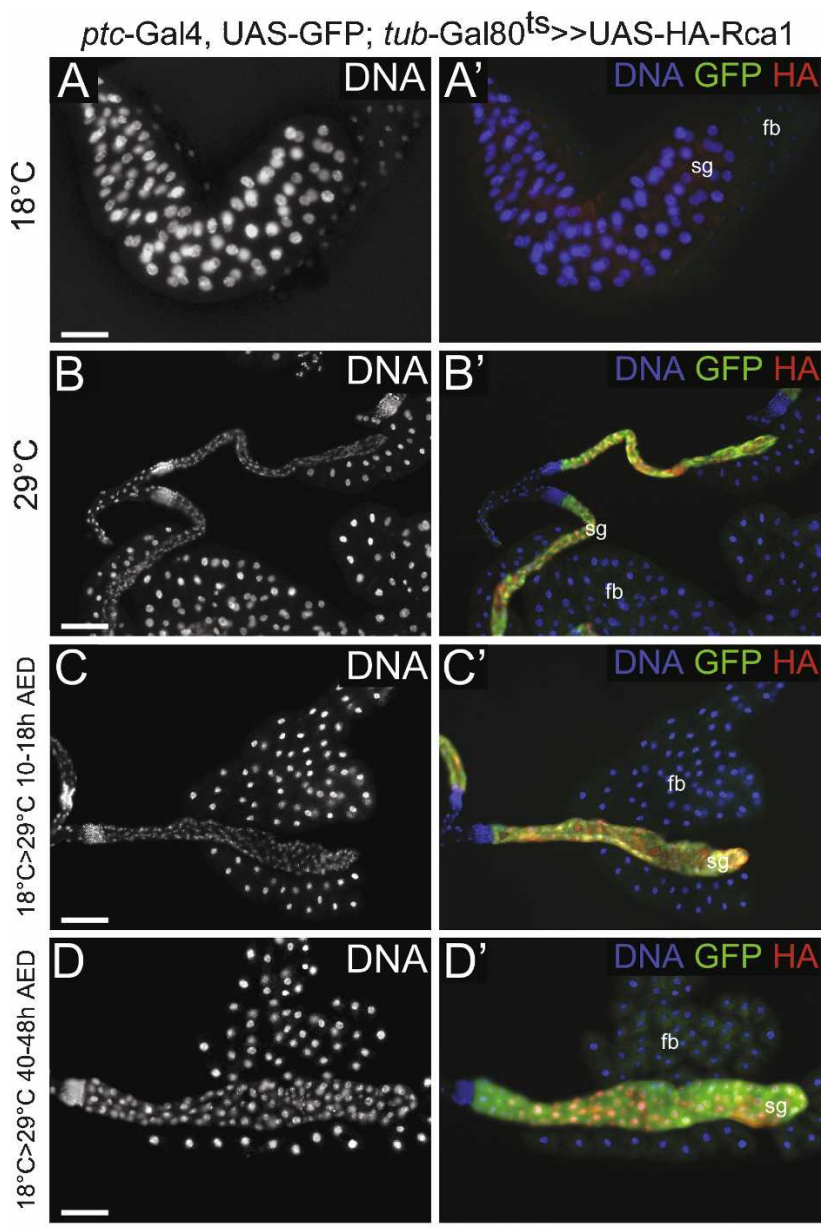

$h s-f l p^{1.22} ;$ act $<\mathrm{y}^{+}>$Gal4, UAS-GFP>>UAS-HA-Rca1

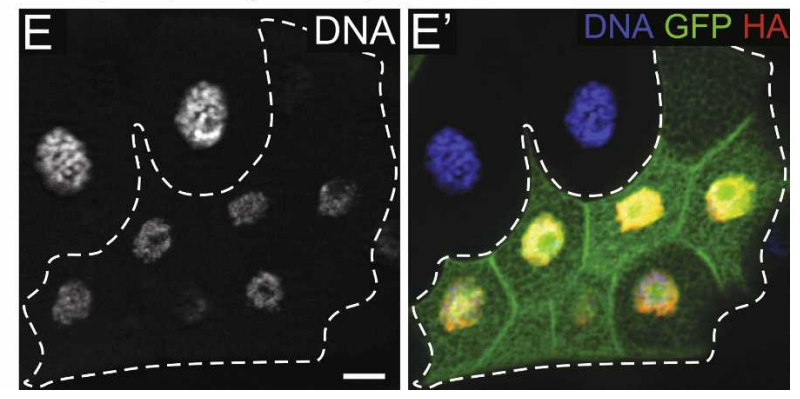

Rca1 misexpression affects CycE levels. We used the flpout method to create salivary glands overexpressing Rcal in individual cells. These mosaic salivary glands contained cells with varying overexpression levels, presumably because of a variable efficiency of removal of the multiple flp-out cassettes from the polytene chromosomes (Fig. 5A). In cells that expressed high amounts of HA-Rcal we observed significantly increased levels of CycE. To investigate whether the elevated CycE protein levels were due to increased transcription, expression of CycE was monitored by a reporter construct that contains $16.4 \mathrm{~kb}$ of the regulatory region of the $\mathrm{CycE}$ gene fused to lacZ (Jones et al. 2000). This reporter construct was highly activated in the Rca1-positive cells (Fig. 5B), indicating that ectopic Rcal expression promotes CycE transcription. 


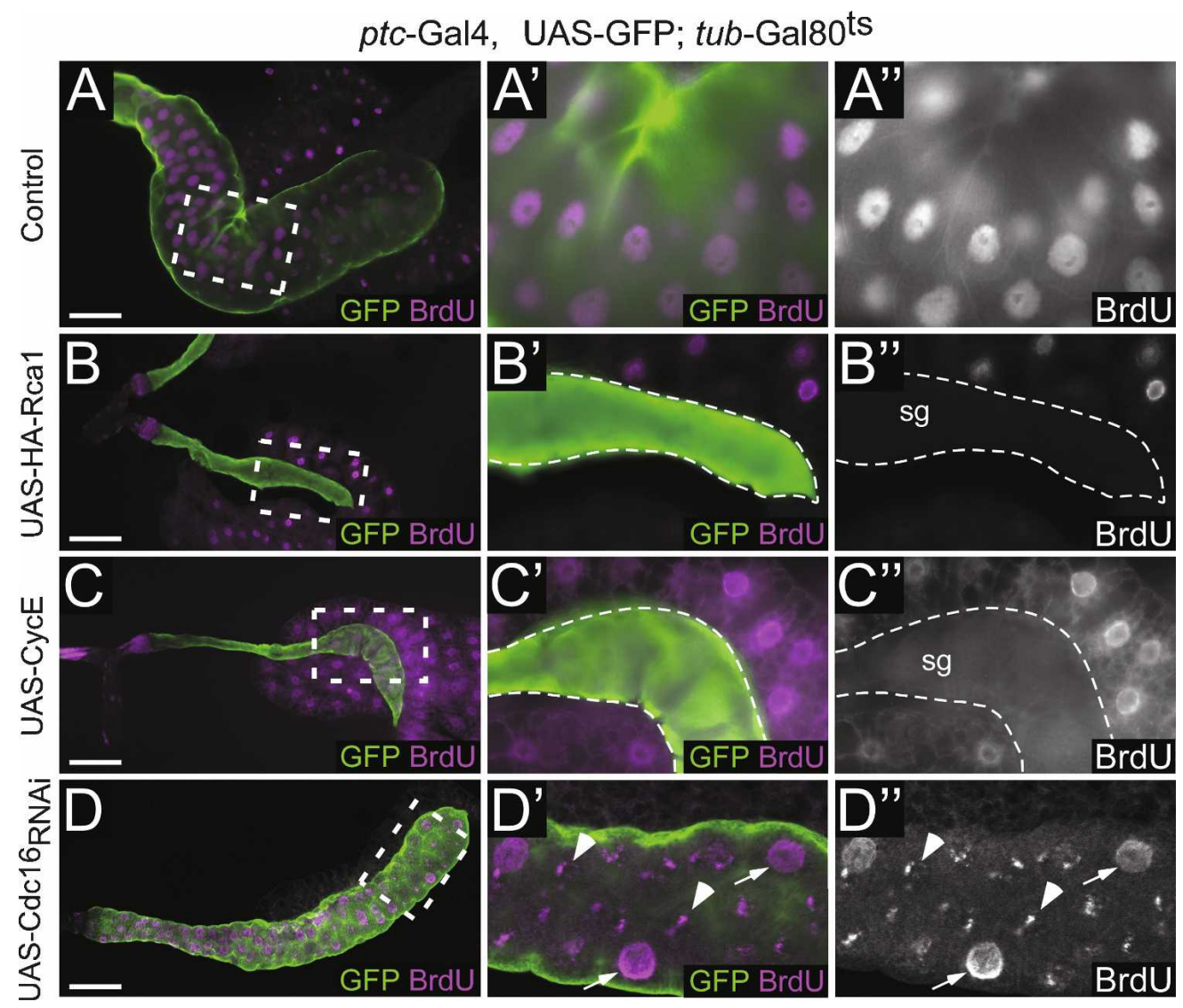

Figure 3. APC/C depletion blocks DNA replication in salivary glands. To visualize DNA replication larvae were fed for $24 \mathrm{~h}$ with BrdU-containing medium. Expression of the indicated constructs was induced 10-18 h AED. BrdU was added to the medium $60 \mathrm{~h}$ post-induction. For conditional expression $p t c-G a l 4$ was applied in combination with $t u b$-Gal80 ${ }^{\text {ts }}$. (A) In wild type, BrdU was detectable throughout the salivary gland, suggesting that all nuclei underwent at least one S phase during the labeling period. $(B)$ Expression of HA-Rcal results in small salivary glands, and no BrdU can be detected within salivary gland nuclei but in adjacent fat body cells, demonstrating that DNA replication has been halted. $(C)$ Overexpression of Cyclin E results in the same phenotype as Rcal overexpression, suggesting that both genes affect similar downstream pathways. $(D) C d c 16$ depletion in salivary glands interferes with endocycle progression. BrdU incorporation is highly reduced in the majority of $C d c 16$-depleted cells. Most cells show a punctuate BrdU pattern that is reminiscent of late DNA replication (arrowheads). However, individual cells were found that appear to undergo normal $\mathrm{S}$ phases (arrows). The weaker phenotype compared with the Rcal overexpression is likely intrinsic to the RNAi technique. Nonetheless, the similarity of the phenotypes suggests that the Rcal overexpression phenotype is due to inhibition of APC/C activity. Bar, $100 \mu \mathrm{m}$.

In mitotic cells CycE and the transcription factor E2F1/Dp cooperate in an autoregulatory feedback loop that ensures accumulation of CycE prior to S-phase entry (Duronio and O'Farrell 1995; Sauer et al. 1995). To determine whether this feedback loop operates in endoreplicating cells, we overexpressed CycE in salivary glands using the flp-out method. This also resulted in up-regulation of the CycE-lacZ reporter construct (Fig. 5C), demonstrating that CycE can stimulate its own expression in endoreplicating salivary glands as well.

To test if this autoregulatory feedback loop relies on the transcription factor E2F1/Dp, we performed qRTPCR on RNA derived from salivary glands in which CycE or HA-Rcal was overexpressed and compared transcript levels with those from wild-type salivary glands (Fig. 5D). We monitored transcript levels of Rca1, CycE, and E2F1, as well as two established E2F1 targets, Rnr2 and PCNA (Duronio et al. 1995; Thacker et al. 2003). This confirmed the marked increase of CycE transcript levels after HA-Rcal expression. Moreover, transcript levels of the E2F1 target genes Rnr2 and PCNA were significantly increased upon overexpression of HA-Rcal or CycE (Fig. 5D), indicating enhanced E2F1 activity in these cells. Interestingly, E2F1 transcript levels were rather reduced after expression of HA-Rcal or CycE (Fig. $5 \mathrm{D})$. The increase in E2F1 target gene expression is therefore not caused by the induction of E2F1 transcription and might rather be a post-transcriptional effect. Consistent with this notion, immunostaining against E2F1 revealed a significant increase of E2F1 protein in cells overexpressing either HA-Rcal or CycE (Fig. 5E,F). The abundance of E2F1 protein is regulated in a cell cycledependent manner. In Drosophila imaginal discs, E2F1 accumulates during G1 but is destroyed during early $S$ 
Figure 4. Compromised APC/C-Fzr activity increases CycE-Cdk2 activity. The MPM-2 antibody recognizes a CycE-Cdk2 phosphorylated protein that assembles into the histone locus body. Conditional overexpression of the indicated constructs was achieved by using ptc-Gal4 together with the TARGET system. Expression was induced 10-18 h AED. (A) In control salivary glands derived from wandering larvae that have terminated endoreplication, CycE activity has ceased and nuclei were devoid of MPM-2 staining. (B) Upon continuous expression of CycE MPM-2-positive subnuclear spheres (filled arrowheads) can be detected in virtually all nuclei of the salivary gland. In addition, brightly stained speckles (open arrowheads), which likely represent heterochromatin, were visible on the DNA. $(C, D)$ MPM-2-positive spheres as well as DNA regions with intense Hoechst staining appear after overexpression of HA-Rcal or knockdown of Cdc16. This indicates that compromised APC/CFzr activity results in increased CycE-Cdk2 activity. It is also important to note that the differences in nuclear size were clearly visible in the high-magnification images. Bar, $10 \mu \mathrm{m}$.

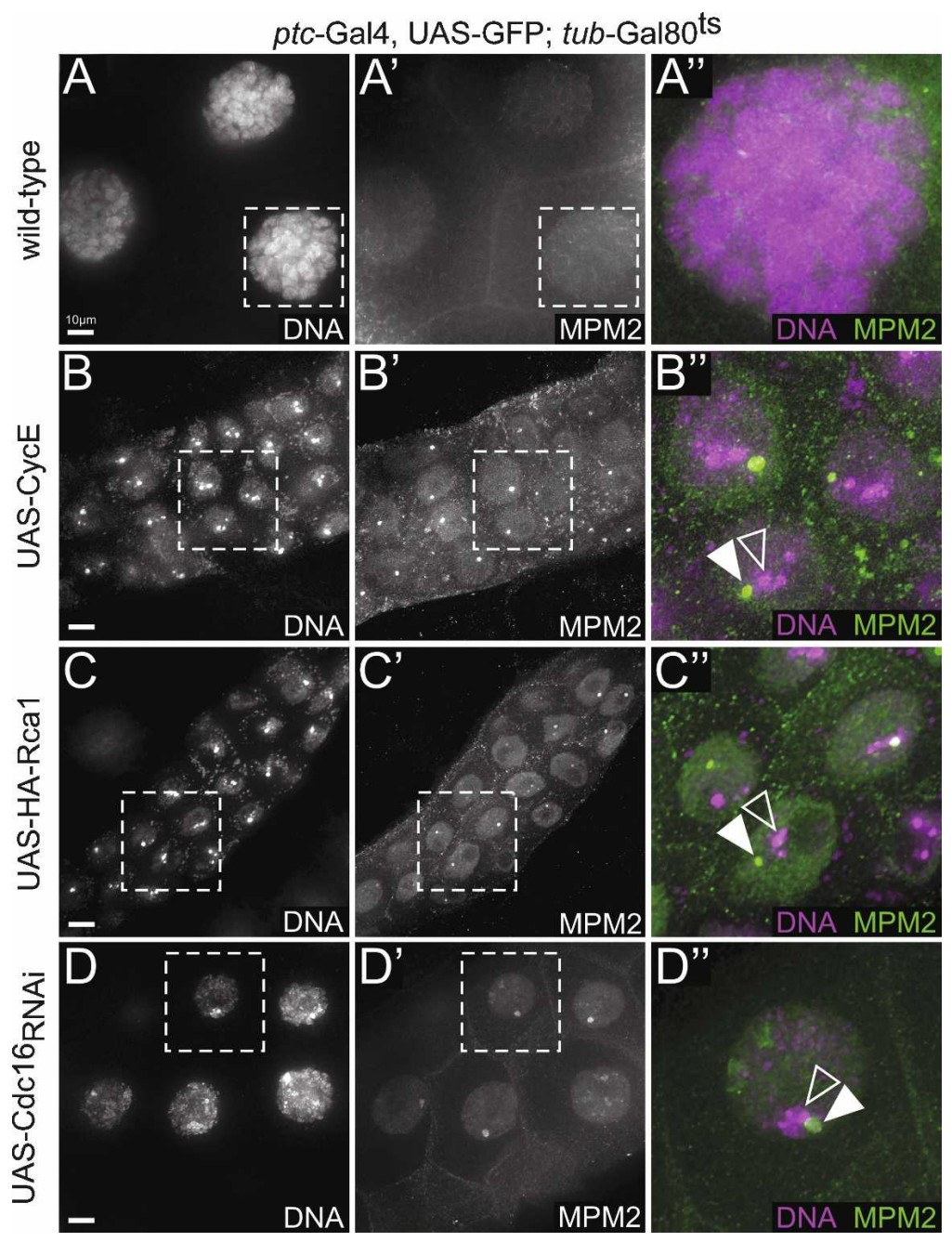

phase (Asano et al. 1996; Heriche et al. 2003; Reis and Edgar 2004). Moreover, it has been recently demonstrated that DNA replication is necessary for E2F1 destruction (Shibutani et al. 2007). Hence, E2F1 stabilization caused by overexpression of HA-Rcal or CycE may be due to the failure to undergo DNA replication, which normally initiates E2F1 turnover. To evaluate this possibility, we inhibited DNA replication by different means and monitored E2F1 protein levels. DNA replication requires the activity of dup, the Drosophila ortholog of Cdt1, which is essential for pre-RC formation (Whittaker et al. 2000; Garner et al. 2001). Geminin is an inhibitor of dup/Cdt1 and overexpression of Geminin can inhibit DNA replication in embryos (Quinn et al. 2001). Overexpression of Geminin in salivary glands using the flp-out technique resulted in small cells with significantly reduced DNA content (Fig. 5G), suggesting that DNA replication was compromised (Fig. 5G). Consistent with the idea that E2F1 turnover is coupled to DNA replication, we found elevated levels of E2F1 (Fig. 5H) and CycE (Fig. 5I) in Geminin-overexpressing cells. Altogether, these results show that E2F1 protein is stabilized when DNA replication is blocked and, probably as a consequence, CycE expression is increased.
Geminin is a critical APC/C target in endoreplicating cells

Our findings demonstrate that inhibition of APC/C activity results in higher E2F1 and CycE levels and interferes with endoreplication. The APC/C controls the abundance of a plethora of proteins, raising the question: Which APC/C target might cause the block of endoreplication? Geminin appeared as a highly attractive candidate because it is involved in the regulation of pre-RC formation during mitotic cycles (McGarry and Kirschner 1998). Immunostaining revealed that Geminin levels were highly elevated in HA-Rcal-overexpressing cells (Fig. 6A). Since ectopic Geminin expression impairs endoreplication, this finding implies that the failure to execute $\mathrm{S}$ phase caused by APC/C-Fzr inactivation is likely due to accumulation of Geminin protein. Studies on Drosophila embryos and human cancer cell lines have demonstrated that APC/C-Fzr is negatively regulated by CycE-Cdk2 (Sigrist and Lehner 1997; Grosskortenhaus and Sprenger 2002; Reber et al. 2006; Keck et al. 2007). Therefore, we tested if overexpression of CycE also results in accumulation of Geminin protein. CycE-overexpressing cells indeed displayed high 

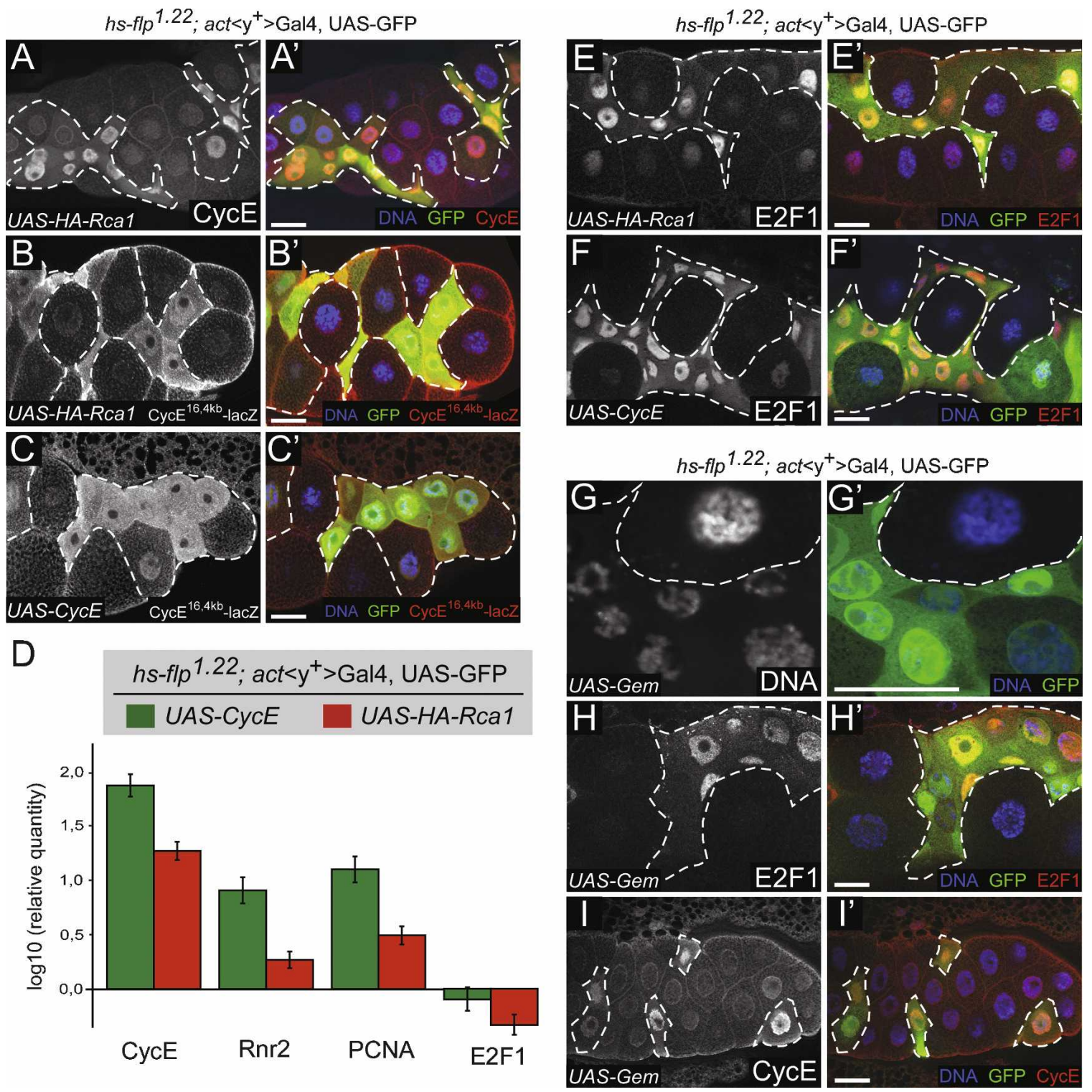

Figure 5. Rcal overexpression results in E2F1 accumulation and subsequent activation of its target genes. The indicated UAS constructs were overexpressed in individual salivary gland cells using the flp-out method. Expression was induced 40-48 h AED. (A) Overexpression of HA-Rcal results in higher levels of CycE protein. $(B)$ A reporter construct comprised of the CycE regulatory region fused to the lacZ gene (CycE ${ }^{16,4 k b}-1 \mathrm{lacZ}$ ) shows that $\mathrm{CycE}$ transcription is enhanced after HA-Rcal overexpression. (C) The CycE-lacZ reporter construct is also up-regulated after $\mathrm{CycE}$ overexpression, indicating the presence an autoregulatory feedback loop. $(D) \mathrm{qRT}-$ PCR reveals that E2F1 target genes were up-regulated in salivary glands overexpressing either CycE or HA-Rca1. Data obtained from wild-type salivary glands was set as a reference point and the relative quantity is shown as a $\log 10$. Transcript levels of CycE, Rnr2, and PCNA were up-regulated after overexpression of HA-Rcal or CycE, while E2F1 transcript levels were not increased. $(E, F)$ E2F1 protein levels were up-regulated in cells overexpressing either HA-Rcal or CycE, indicating that CycE accumulation is due to increased E2F1-dependent transcription. $(G)$ Overexpression of Geminin disturbs endocycle progression. The GFP-marked cells in which Geminin was overexpressed displayed a markedly reduced DNA content compared with neighboring control nuclei. $(H, I)$ Overexpression of Geminin results in elevated levels of E2F1 and CycE protein, demonstrating that E2F1 and its target genes generally accumulate in cells with impaired DNA replication. Bar, $50 \mu \mathrm{m}$.

levels of Geminin (Fig. 6B), thus providing an explanation for the similarity of the CycE and Rcal overexpression phenotypes. In summary, inactivation of APC/CFzr results in accumulation of Geminin and perturbs DNA endoreplication. This is associated with the stabilization of E2F1 protein and enhanced expression of its target genes, including CycE. Increased CycE-Cdk2 activity enhances the inhibition of the APC/C-Fzr complex (Sigrist and Lehner 1997; Grosskortenhaus and Sprenger 2002; Reber et al. 2006; Keck et al. 2007) and may amplify the negative effect by increasing Geminin stability. However, we cannot exclude that CycE-Cdk2 

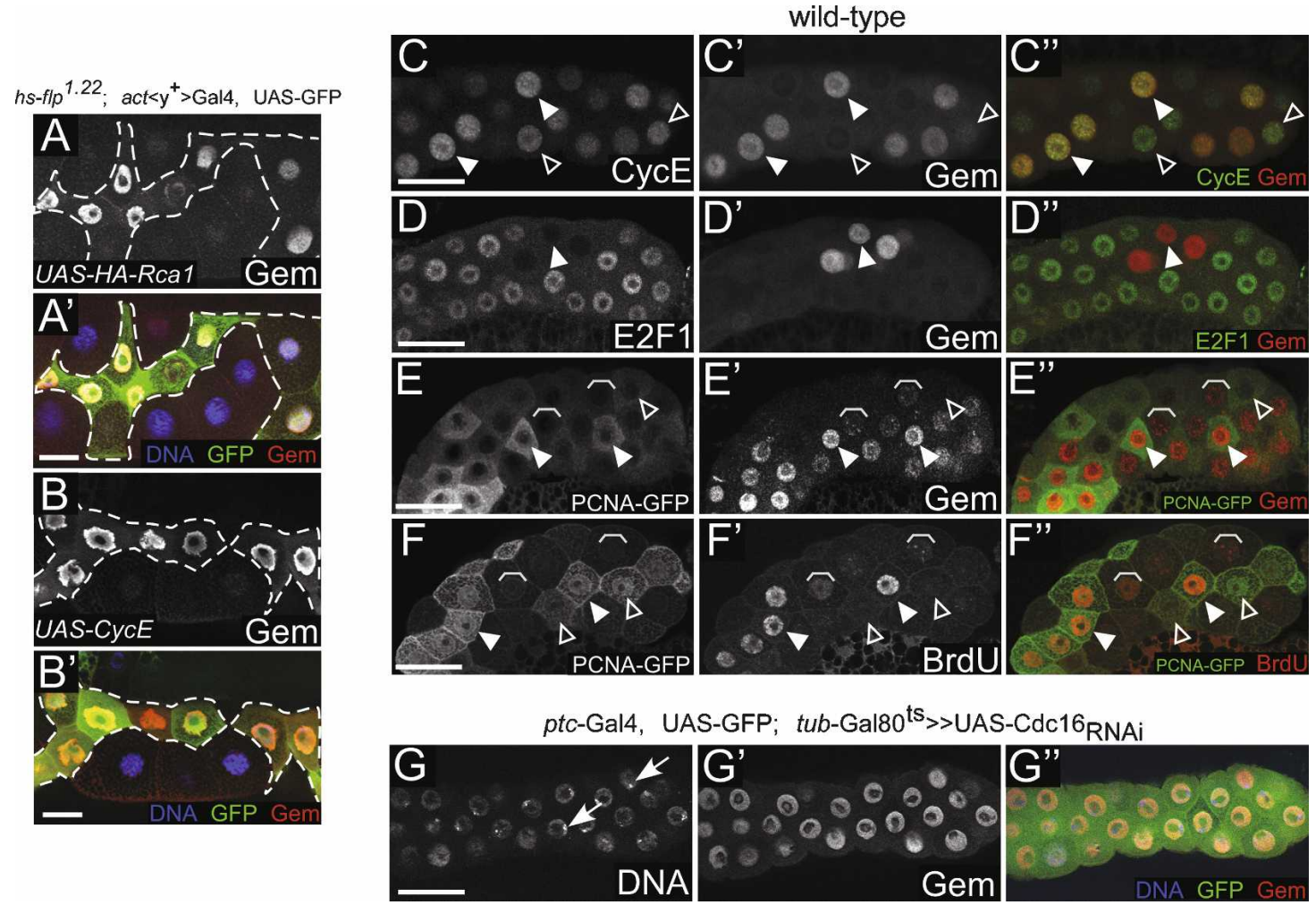

Figure 6. APC/C-Fzr activity is crucial for Geminin oscillation during endoreplication. $(A, B)$ Geminin is up-regulated after overexpression of HA-Rcal or CycE. Individual cells overexpressing HA-Rcal or CycE were generated by the flp-out technique 40-48 h AED. $(C-F)$ Geminin protein levels fluctuate during salivary gland endocycles. Wild-type salivary glands derived from early third instar larvae (72-74 h AED) were stained with antibodies against Geminin and either CycE $(C)$, E2F1 $(D)$, or PCNA-GFP $(E)$. As a reference, PCNA-GFP-expressing salivary glands of the same age were labeled for $1 \mathrm{~h}$ with $\mathrm{BrdU}(F)$. Costaining with these cell cycle markers allowed us to categorize individual cells into G phase, late G phase/early S phase (open arrowheads), mid-S phase (closed arrowheads), or late S phase (brackets). Cells in G phase display high levels of E2F1 protein. Late G-phase/early S-phase cells are positive for PCNA-GFP, contain moderate levels of CycE but are negative for BrdU. Mid-S-phase cells show high levels of BrdU, CycE, and PCNA-GFP, but lack E2F1 protein. In late S-phase cells, PCNA-GFP is only present at trace amounts and only residual levels of BrdU were visible. Geminin can be detected in mid to late S-phase cells. (F) RNAi-mediated knockdown of Cdc16 in salivary glands results in stabilization of Geminin protein levels in all cells throughout the salivary gland. The appearance of brightly stained DNA regions (arrows) indicates that Cdc16 depletion was effective. Expression of Cdc16-RNAi was induced 10-18 h AED. Conditional expression was achieved by using $p t c$-Gal4 together with the TARGET system. Bar, $50 \mu \mathrm{m}$.

activity inhibits pre-RC assembly by additional mechanisms.

In wild-type salivary glands dissected from feeding larvae, Geminin is only detectable in a subset of cells (Fig. $6 \mathrm{C}-\mathrm{E})$, suggesting that Geminin protein oscillates during endoreplication. In order to determine the cell cycle stage in which Geminin protein accumulates, we performed double labelings of Geminin and either CycE, E2F1, or PCNA-GFP. CycE protein accumulates at the end of $\mathrm{G}$ phase and persists throughout $S$ phase (Weng et al. 2003). By contrast, E2F1 protein is only present in G-phase cells and gets degraded upon initiation of DNA replication (V.K. Tran and B.A. Edgar, pers. comm.). Geminin protein was only detectable in cells exhibiting high CycE levels (Fig. 6C). This is consistent with the idea that Geminin accumulation requires inhibition of APC/C-Fzr activity by CycE-Cdk2. Conversely, Geminin protein was absent in cells with high E2F1 levels
(Fig. 6D). Altogether, these experiments indicate that Geminin accumulates in S-phase cells, but gets degraded once cells have completed DNA replication. To visualize cells with ongoing DNA replication we used the PCNAGFP reporter, which is activated in S-phase cells (Duronio et al. 1995; Thacker et al. 2003). We compared PCNA-GFP with BrdU incorporation and PCNA-GFP with Geminin protein levels (Fig. 6E,F). Mid-S-phase cells are BrdU-positive and display high PCNA-GFP staining. In these cells (Fig. 6E,F, marked with filled arrowheads) Geminin is highly expressed. Cells in late G phase/early $S$ phase (Fig. 6E,F, open arrowheads) display PCNA-GFP staining but are yet not positive for BrdU. In these cells, Geminin is absent or only weakly detectable. Cells in late $S$ phase are characterized by diminished PCNA-GFP staining and weak BrdU incorporation (Fig. $6 \mathrm{E}, \mathrm{F}$, brackets). In cells of this category Geminin is already reduced. These data show that Geminin accumu- 
lates when cells enter $S$ phase and gets degraded upon exit from $S$ phase to allow relicencing of replication origins. Since Geminin is an APC/C-Fzr target (Supplemental Fig. S1F; McGarry and Kirschner 1998) its oscillation might be mediated by fluctuations in APC/C-Fzr activity. To test this idea we inactivated APC/C throughout the salivary gland by overexpressing Cdc16-RNAi using $p t c$-Gal4. Strikingly, Geminin protein was detectable in all cells of the salivary gland (Fig. 6F), demonstrating that APC/C-Fzr activity is crucial for Geminin oscillation during endoreplication.

\section{Discussion}

In this study we show that APC/C-Fzr activity is essential for endocycle progression. Our data suggests that the APC/C-Fzr complex regulates the abundance of the Geminin protein during salivary gland endoreplication cycles, and that this is important to prevent relicensing in $\mathrm{S}$ phase. Inactivation of the APC/C-Fzr complex in larval salivary glands is accompanied by impaired DNA replication and stabilization of Geminin protein. Consistent with this observation recent work demonstrated that giant trophoblast cells in Fzr knockout mice fail to undergo endoreplication (I. Garcia-Higuera, M. Malumbres, and S. Moreno, pers. comm.). Our findings about the requirement of the APC/C during endoreplication cycles are consistent with previous studies on $m r / A P C 2$ mutants that display small salivary glands with thin polytene chromosomes (Reed and Orr-Weaver 1997). Furthermore, our results are in agreement with a recent publication that independently demonstrates that depletion of APC/C activity compromises endoreplication in larval salivary glands (Narbonne-Reveau et al. 2008). It has been proposed that the endocycle is driven by oscillating activities of the transcription factor E2F1 and $\mathrm{CycE}$, which acts in concert with its designated kinase partner Cdk2 (Edgar and Nijhout 2004). In agreement with the model suggested by Edgar and Nijhout, we found that E2F1 and CycE levels fluctuate in endoreplicating in salivary glands. Moreover, double-labeling experiments with BrdU have revealed that E2F1 is only abundant in cells that reside in G phase, but absent in cells with ongoing DNA replication (V.K. Tran and B.A. Edgar, pers. comm.). CycE displays a complementary pattern since it accumulates in late $\mathrm{G}$ phase and persists throughout $S$ phase (Weng et al. 2003). These observations are consistent with a model in which E2F1 and its cofactor $\mathrm{dDP}$ activate the transcription of CycE during late G1 phase. CycE-Cdk2 then enhances its own activity by inhibiting the E2F1 repressor Rbf (Du et al. 1996) resulting in a sharp peak of Cdk2 activity, which eventually triggers S-phase entry. The onset of DNA replication in turn initiates a negative feedback loop that downregulates E2F1 activity and simultaneously triggers dup/ Cdt1 degradation (May et al. 2005). The short half-live of CycE mRNA and protein would then suffice to cause a drop in CycE-Cdk2 activity after completion of DNA replication (Fig. 7).
CycE-Cdk2 regulates APC/C-Fzr activity in salivary glands

Three pathways tightly control APC/C-Fzr activity during the mitotic cycles of Drosophila embryogenesis. The best characterized inhibitor of the APC/C-Fzr complex is Rcal, the Drosophila ortholog of the vertebrate Emil proteins (Grosskortenhaus and Sprenger 2002). In addition, it has been demonstrated that CycA-Cdk1 and CycE-Cdk2 complexes also contribute to APC/C-Fzr inhibition during Drosophila embryogenesis (Sigrist and Lehner 1997; Grosskortenhaus and Sprenger 2002; Dienemann and Sprenger 2004; Reber et al. 2006). However, expression profiling showed that CycA, Cdk1, and Rcal are only weakly expressed in larval salivary glands, indicating that these genes are presumably not involved in APC/C-Fzr regulation during endoreplication. Moreover, we found no obvious phenotype in salivary gland cells mutant for rca1. The result that Rcal activity is required in mitotic but not in endoreplicating cells is consistent with the phenotype of Emil-deficient mice, in which the mitotic cells of the inner cell mass cells die, whereas the endoreplicating trophoblast giant cells appear normal (Lee et al. 2006). Therefore, CycE-Cdk2 is the only known APC/C-Fzr inhibitor in larval salivary glands. Double-labeling experiments on endoreplicating salivary glands revealed that Geminin is only abundant in cells with high $\mathrm{CycE}-\mathrm{Cdk} 2$ activity. Furthermore, we found that Geminin protein accumulates in salivary gland cells that continuously overexpress CycE from a transgene. The idea that the APC/C-Fzr complex is negatively regulated by $\mathrm{CycE}$-dependent kinase activity has recently been corroborated by biochemical data, which showed that human CycE-Cdk2 can phosphorylate Cdh1/Fzr and thereby promote its dissociation from the APC/C core complex (Keck et al. 2007). The unique function of Cyclin E during endoreplication is emphasized by the finding that trophoblast giant cells and megakaryocytes in Cyclin E-deficient mice fail to undergo endoreplication, whereas mitotic cells proliferate rather normally (Geng et al. 2003; Parisi et al. 2003). These observations have substantial implications for the current view of endoreplication. We propose that $\mathrm{CycE} /$ Cdk2-mediated inhibition of APC/C activity ensures that the peak of CycE-dependent kinase activity simultaneously initiates DNA replication, and prevents relicensing through stabilization of Geminin. In the following $\mathrm{G}$ phase, when CycE-Cdk2 activity is low, the APC/ C-Fzr complex is released from this inhibition and targets Geminin for proteasomal degradation, allowing licensing of replication origins (Fig. 7).

\section{Geminin is important to prevent rereplication in endocycle $S$ phases}

In the endoreplicating tissues of geminin mutant embryos massive overreplication has been observed, demonstrating that Geminin activity is required to limit DNA replication during endoreplication (Quinn et al. 2001). Conversely, we found that endoreplication is se- 


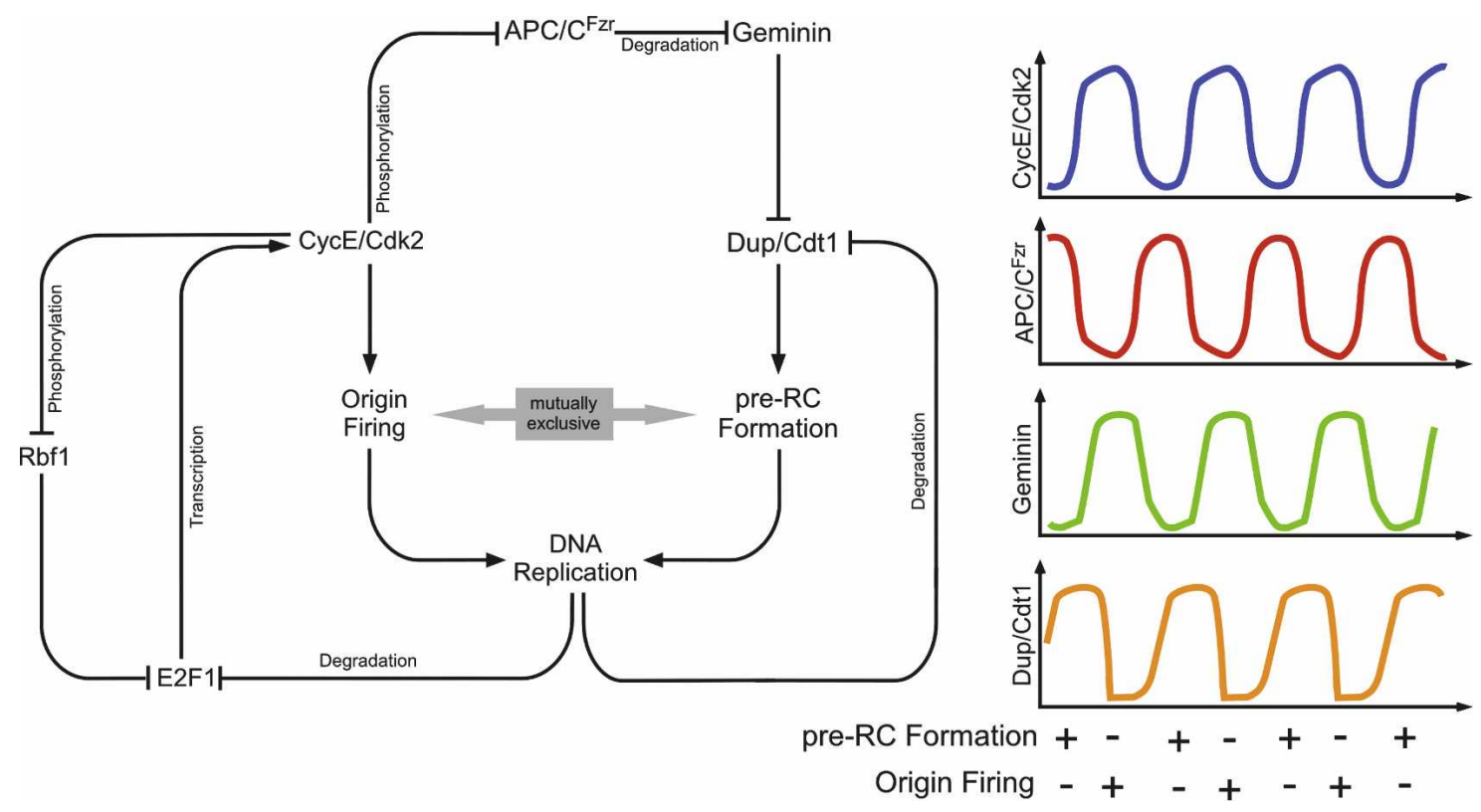

Figure 7. Oscillating APC/C-Fzr activity is essential for rereplication control in endoreplicating cells. The endocycle is driven by oscillating activities of CycE-Cdk2 and the transcription factor E2F1. During G phases, CycE transcription is activated by E2F1. CycE-Cdk2 enhances its own activity by inhibiting the E2F1 repressor Rbf. This peak of CycE-dependent kinase activity eventually triggers S-phase entry and simultaneously inhibits APC/C-Fzr activity allowing accumulation of Geminin protein. High levels of Geminin prevent relicensing by sequestering dup/Cdt1, which is an essential factor for the assembly of the pre-RC. The initiation of DNA replication activates a negative feedback loop that down-regulates E2F1 activity and simultaneously promotes the degradation of dup/Cdt1. The subsequent decrease of CycE-Cdk2 activity releases the APC/C-Fzr complex, which in turn mediates Geminin degradation. This enables dup/Cdt1 to promote pre-RC formation during the next $\mathrm{G}$ phase, thereby preparing the next $\mathrm{S}$ phase. Thus, this mechanism allows temporal separation of licensing and firing of replication origins, two processes that are mutually exclusive.

verely impaired in salivary gland cells forced to overexpress Geminin. This suggests that the impaired endoreplication observed in cells overexpressing either Rcal or CycE results from elevated Geminin levels. However, it cannot be excluded that other APC/C-regulated proteins, besides Geminin, contribute to the endocycle arrest observed upon APC/C inactivation. A recent study on Geminin-deficient mice revealed that cells lacking Geminin have higher DNA contents than wild-type cells and therefore the investigators suggested that loss of Geminin commits mitotic cells to endoreplication (Gonzalez et al. 2006). This hypothesis contradicts our model in which Geminin is essential for rereplication control during endoreplication. However, no flow cytometric analysis was included in this study to confirm that Geminin mutant mouse cells underwent true endocycles with distinct $\mathrm{G}$ phases, rather than overreplicating their DNA continuously due to the lack of proper licencing control. Consistent with our finding that Geminin accumulates upon inactivation of the APC/CFzr complex, Gonzalez et al. reported that treatment of mouse embryos with a proteasome inhibitor led to the stabilization of Geminin protein. The investigators of this study claimed that the APC/C-Fzr complex is constitutively activated in endoreplicating cells, and that constant degradation of Geminin is part of the normal mechanism of endocycle regulation. However, we could demonstrate that Geminin is present in endoreplicating cells of the salivary gland. In agreement with its established function to control DNA licencing, we found that Geminin is present during $S$ phase but absence in $G$ phase. These oscillations are likely caused by periodic inhibition of APC/C-Fzr activity by CycE-Cdk2. Thus, we propose that the fluctuation of Geminin protein is crucial for the control DNA licensing during endoreplication. Futhermore, we suggest that similar oscillations of APC/C activity and Geminin levels also occur in endoreplicating cells of mouse embryos.

\section{Materials and methods}

Fly stocks

$\mathrm{W}^{1118}$ flies were used as a control and further on referred to as wild type. Strains carrying UAS-RNAi constructs were obtained from the RNAi collection at the National Institute for Genetics (Mishima, Shizuoka, Japan): UAS-Cdc16-RNAi (6759R-3) and UAS-Rca1-RNAi (10800R-1, 10800R-2). Mutant or transgene fly stocks that were described elsewhere: w; Frt40A, rca1 ${ }^{2}$ / $\mathrm{CyO}_{\text {wg-lacz }}$, and UAS-HA-Rcal (Grosskortenhaus and Sprenger 2002); UAS-CycE (Sigrist and Lehner 1997); UAS-Geminin (Quinn et al. 2001); CycE-lacZ ${ }^{16,4 k b}$ (Jones et al. 2000; Wu et al. 2003); PCNA-GFP (Thacker et al. 2003); en-Gal4; UAS-GFP/ SM6-TM6 (Neufeld et al. 1998); hh-Gal4 (Tanimoto et al. 2000); GFP-Cdc16 (Huang and Raff 2002). Stocks used for clonal analysis and conditional expression of UAS constructs were kindly provided by Thomas Klein (University of Cologne, Germany): 
w, hs-flp ${ }^{1.22}$; act $<\mathrm{y}^{+}>$Gal4(25), UAS-GFP/SM6-TM6; w, hs-flp ${ }^{1.22}$, tub-Gal4, UAS-GFP/FM7; Frt40A, Gal80/CyO, and w; ptc-Gal4, UAS-GFP; tub-Gal80 ${ }^{\text {ts }} /$ TM6B

\section{Fly breeding}

Wild-type larvae were collected on grape juice plates and staged to $72-74 \mathrm{~h}$ AED at $25^{\circ} \mathrm{C}$. The TARGET system and ptc-Gal4 was used for conditional overexpression of UAS constructs in salivary glands (McGuire et al. 2004; Pierce et al. 2004). Embryos were collected for $18 \mathrm{~h}$ at $18^{\circ} \mathrm{C}$ and aged then for another $22 \mathrm{~h} / 88 \mathrm{~h}$ at $18^{\circ} \mathrm{C}$. Expression in salivary glands was induced by shifting temperature to $29^{\circ} \mathrm{C}$. Samples were raised at $29^{\circ} \mathrm{C}$ until larvae initiated wandering, and were then immediately used for immunohistochemistry. Drosophila development is accelerated by cultivation at $29^{\circ} \mathrm{C}$, whereas it is retarded at $18^{\circ} \mathrm{C}$. Therefore, all incubation times were normalized to developmental timing at $25^{\circ} \mathrm{C}$ according to Buttitta et al. (2007). Individual salivary gland cells overexpressing certain UAS constructs were generated by the flp-out technique (Ito et al. 1997). Expression was induced $40-48 \mathrm{~h}$ by a $10-\mathrm{min}$ heat shock at $37^{\circ} \mathrm{C}$. Loss of function clones in the salivary placode were created with the MARCM technique (Lee and Luo 1999). Embryos were collected for $8 \mathrm{~h}$ at $25^{\circ} \mathrm{C}$ and subsequently heat-shocked for $1 \mathrm{~h}$ at $37^{\circ} \mathrm{C}$. Both MARCM and flp-out experiments were cultivated at $25^{\circ} \mathrm{C}$ until larvae initiated wandering and subsequently analyzed by immunohistochemistry. To determine depletion efficiencies, UAS-RNAi constructs were continuously overexpressed in wing imaginal discs using either en-Gal4 or hh-Gal4. In this case, flies were cultivated at $29^{\circ} \mathrm{C}$ to achieve maximum expression strength with the UAS/Gal4 system. Detailed information about temporal parameters of the experiments can be found in the Supplemental Material.

\section{Immunohistochemistry}

Salivary glands were dissected in PBS and fixed for $30 \mathrm{~min}$ at $24^{\circ} \mathrm{C}$ in $4 \%$ paraformaldehyde/PBS. Isolated tissues were blocked for $1 \mathrm{~h}$ at $24^{\circ} \mathrm{C}$ in PBS/0.3\% Triton X-100/5\% NGS. Primary antibodies were used in the following dilutions: rat anti-HA (1:100; Roche), rat anti-Geminin (1:500) (Quinn et al. $2001)$, guinea pig anti-CycE (1:500 to $1: 800$; gift of T. OrrWeaver, Whitehead Institute, Cambridge, MA), guinea pig antiE2F1 (1:500, T. Orr-Weaver); rabbit anti-GFP (1:500; Torrey Pines Biolabs); mouse anti-MPM-2 (1:200; Millipore), and mouse anti-BrdU (1:20; Becton Dickinson). Secondary antibodies, purchased from Invitrogen, were used at a dilution of 1:500. DNA was visualized with Hoechst $33258(0.5 \mathrm{mg} / \mathrm{mL}$; Sigma) diluted 1:2000. For BrdU labeling, $200 \mu \mathrm{g} / \mathrm{mL}$ BrdU (Sigma) in $20 \%$ sucrose/PBS was added to the medium $60 \mathrm{~h}$ after induction of genes expression. After $20 \mathrm{~h}$ of incubation at $29^{\circ} \mathrm{C}$, salivary glands were isolated and processed according to Baker and $\mathrm{Yu}$ (2001). Samples were generally mounted in Vectashield (Vector Laboratories).

\section{Image acquisition and processing}

Epifluorescence images of whole salivary glands were taken on a Zeiss AxioImager fitted with a CCD camera (Zeiss AxioCam MRc5). Higher resolution images represent single confocal sections that were acquired either on a Zeiss AxioImager fluorescent microscope equipped with an Apotome slider module (Zeiss) or on a Leica TCS-SP2 laser scanning microscope. Highresolution images of salivary gland nuclei shown in Figure 5 were taken on a Deltavision RT imaging system (Applied precision). Z-stacks of 200- $\mu \mathrm{m}$ sections were captured using a $60 \times$,
NA 1.4 Oil-Objective (Olympus) and deconvolved (20 iterations in the hard-aggressive mode) with the SoftWorx Imaging Suite (Applied Precision). Maximum projections encompassing whole salivary gland nuclei were generated with $\mathrm{NIH}$ ImageJ. Adobe Photoshop CS2 and ACD Canvas X software were used for image assembly.

\section{Quantitative real-time PCR}

Detailed information about the qRT-PCR analysis can be found in the Supplemental Material.

\section{Western blotting}

Extracts containing different amounts of wild-type embryos or larval salivary glands were separated by SPS-PAGE and transferred to nitrocellulose membranes according to standard methods. Blotted membranes were stained with Ponceau S and then probed with following primary antibodies: rabbit anti-Fzr (1:20) (Jacobs et al. 2002); mouse anti-tubulin (1:3000; Sigma). Appropriate HRP-coupled secondary antibodies purchased from Jackson Immunolabs were diluted 1:1000. Proteins were visualized using the ECL-Western blotting detection system (Amersham).

\section{Acknowledgments}

N.Z. is grateful to Bruce A. Edgar for communication of unpublished results and the allowance to complete this work in his laboratory. We also acknowledge T. Orr-Weaver, H. Richardson, M. Asano, R. Duronio, T. Klein, and the National Institute of Genetics (Japan) for providing reagents, as well as M. Zigman for critical reading of the manuscript. Finally, we thank S. Moreno for the permission to discuss data prior to publication. This work was partly funded by a post-doctoral fellowship from the German Academic Exchange Service (DAAD) to N.Z., NIH grant GM51186 to B.A.E., and by the Deutsche Forschungsgemeinschaft through SFB grant 572 to F.S.

\section{References}

Arias, E.E. and Walter, J.C. 2007. Strength in numbers: Preventing rereplication via multiple mechanisms in eukaryotic cells. Genes \& Dev. 21: 497-518.

Asano, M., Nevins, J.R., and Wharton, R.P. 1996. Ectopic E2F expression induces $\mathrm{S}$ phase and apoptosis in Drosophila imaginal discs. Genes \& Dev. 10: 1422-1432.

Baker, N.E. and Yu, S.Y. 2001. The EGF receptor defines domains of cell cycle progression and survival to regulate cell number in the developing Drosophila eye. Cell 104: 699708

Bell, S.P. and Dutta, A. 2002. DNA replication in eukaryotic cells. Annu. Rev. Biochem. 71: 333-374.

Blow, J.J. and Dutta, A. 2005. Preventing re-replication of chromosomal DNA. Nat. Rev. Mol. Cell Biol. 6: 476-486.

Buttitta, L.A., Katzaroff, A.J., Perez, C.L., de la Cruz, A., and Edgar, B.A. 2007. A double-assurance mechanism controls cell cycle exit upon terminal differentiation in Drosophila. Dev. Cell 12: 631-643.

Calvi, B.R., Lilly, M.A., and Spradling, A.C. 1998. Cell cycle control of chorion gene amplification. Genes \& Dev. 12: 734-744.

de Nooij, J.C., Graber, K.H., and Hariharan, I.K. 2000. Expression of the cyclin-dependent kinase inhibitor Dacapo is regulated by cyclin E. Mech. Dev. 97: 73-83.

Deak, P., Donaldson, M., and Glover, D.M. 2003. Mutations in 
makos, a Drosophila gene encoding the Cdc27 subunit of the anaphase promoting complex, enhance centrosomal defects in polo and are suppressed by mutations in twins/aar, which encodes a regulatory subunit of PP2A. J. Cell Sci. 116: 41474158.

Di Fiore, B. and Pines, J. 2007. Emil is needed to couple DNA replication with mitosis but does not regulate activation of the mitotic APC/C. J. Cell Biol. 177: 425-437.

Dienemann, A. and Sprenger, F. 2004. Requirements of cyclin a for mitosis are independent of its subcellular localization. Curr. Biol. 14: 1117-1123.

Du, W., Vidal, M., Xie, J.E., and Dyson, N. 1996. RBF, a novel RB-related gene that regulates E2F activity and interacts with cyclin E in Drosophila. Genes \& Dev. 10: 1206-1218.

Duronio, R.J. and O'Farrell, P.H. 1995. Developmental control of the G1 to S transition in Drosophila: Cyclin Eis a limiting downstream target of E2F. Genes \& Dev. 9: 1456-1468.

Duronio, R.J., O'Farrell, P.H., Xie, J.E., Brook, A., and Dyson, N. 1995. The transcription factor E2F is required for $S$ phase during Drosophila embryogenesis. Genes \& Dev. 9: 14451455.

Edgar, B.A. and Nijhout, H.F. 2004. Growth and cell cycle control in Drosophila. In Cell growth-Control of cell size (eds. M.N. Hall et al.), pp. 23-83. CSHL Press, Cold Spring Harbor, NY.

Edgar, B.A. and Orr-Weaver, T.L. 2001. Endoreplication cell cycles: More for less. Cell 105: 297-306.

Follette, P.J., Duronio, R.J., and O'Farrell, P.H. 1998. Fluctuations in cyclin $\mathrm{E}$ levels are required for multiple rounds of endocycle S phase in Drosophila. Curr. Biol. 8: 235-238.

Garner, M., van Kreeveld, S., and Su, T.T. 2001. mei-41 and bub1 block mitosis at two distinct steps in response to incomplete DNA replication in Drosophila embryos. Curr. Biol. 11: 1595-1599.

Geng, Y., Yu, Q., Sicinska, E., Das, M., Schneider, J.E., Bhattacharya, S., Rideout, W.M., Bronson, R.T., Gardner, H., and Sicinski, P. 2003. Cyclin E ablation in the mouse. Cell 114: 431-443.

Gonzalez, M.A., Tachibana, K.E., Adams, D.J., van der Weyden, L., Hemberger, M., Coleman, N., Bradley, A., and Laskey, R.A. 2006. Geminin is essential to prevent endoreduplication and to form pluripotent cells during mammalian development. Genes \& Dev. 20: 1880-1884.

Grosskortenhaus, R. and Sprenger, F. 2002. Rcal inhibits APC$\mathrm{Cdh} 1(\mathrm{Fzr})$ and is required to prevent cyclin degradation in G2. Dev. Cell 2: 29-40.

Heriche, J.K., Ang, D., Bier, E., and O'Farrell, P.H. 2003. Involvement of an SCFSlmb complex in timely elimination of E2F upon initiation of DNA replication in Drosophila. BMC Genet. 4: 9. doi: 10.1186/1471-2156-4-9.

Huang, J.Y. and Raff, J.W. 2002. The dynamic localisation of the Drosophila APC/C: Evidence for the existence of multiple complexes that perform distinct functions and are differentially localised. J. Cell Sci. 115: 2847-2856.

Ito, K., Awano, W., Suzuki, K., Hiromi, Y., and Yamamoto, D. 1997. The Drosophila mushroom body is a quadruple structure of clonal units each of which contains a virtually identical set of neurones and glial cells. Development 124: 761771.

Jacobs, H., Richter, D., Venkatesh, T., and Lehner, C. 2002. Completion of mitosis requires neither fzr/rap nor fzr2, a male germline-specific Drosophila Cdh1 homolog. Curr. Biol. 12: 1435-1441.

Jones, L., Richardson, H., and Saint, R. 2000. Tissue-specific regulation of cyclin E transcription during Drosophila melanogaster embryogenesis. Development 127: 4619-4630.
Kashevsky, H., Wallace, J.A., Reed, B.H., Lai, C., Hayashi-Hagihara, A., and Orr-Weaver, T.L. 2002. The anaphase promoting complex/cyclosome is required during development for modified cell cycles. Proc. Natl. Acad. Sci. 99: 11217-11222.

Keck, J.M., Summers, M.K., Tedesco, D., Ekholm-Reed, S., Chuang, L.C., Jackson, P.K., and Reed, S.I. 2007. Cyclin E overexpression impairs progression through mitosis by inhibiting APCCdh1. J. Cell Biol. 178: 371-385.

Klebes, A., Biehs, B., Cifuentes, F., and Kornberg, T.B. 2002 Expression profiling of Drosophila imaginal discs. Genome Biol. 3: RESEARCH0038.1-RESEARCH0038-16. doi: 10.1186/ gb-2002-3-8-research0038.

Knoblich, J.A., Sauer, K., Jones, L., Richardson, H., Saint, R., and Lehner, C.F. 1994. Cyclin E controls S phase progression and its down-regulation during Drosophila embryogenesis is required for the arrest of cell proliferation. Cell 77: 107-120.

Lane, M.E., Elend, M., Heidmann, D., Herr, A., Marzodko, S., Herzig, A., and Lehner, C.F. 2000. A screen for modifiers of cyclin E function in Drosophila melanogaster identifies Cdk2 mutations, revealing the insignificance of putative phosphorylation sites in Cdk2. Genetics 155: 233-244.

Lee, T. and Luo, L. 1999. Mosaic analysis with a repressible cell marker for studies of gene function in neuronal morphogenesis. Neuron 22: 451-461.

Lee, H., Lee, D.J., Oh, S.P., Park, H.D., Nam, H.H., Kim, J.M., and Lim, D.S. 2006. Mouse emil has an essential function in mitotic progression during early embryogenesis. Mol. Cell. Biol. 26: $5373-5381$.

Lilly, M.A. and Duronio, R.J. 2005. New insights into cell cycle control from the Drosophila endocycle. Oncogene 24: 27652775.

Lilly, M.A. and Spradling, A.C. 1996. The Drosophila endocycle is controlled by Cyclin E and lacks a checkpoint ensuring S-phase completion. Genes \& Dev. 10: 2514-2526.

Machida, Y.J. and Dutta, A. 2007. The APC/C inhibitor, Emil, is essential for prevention of rereplication. Genes \& Dev. 21: 184-194.

Machida, Y.J., Hamlin, J.L., and Dutta, A. 2005. Right place, right time, and only once: Replication initiation in metazoans. Cell 123: 13-24.

May, N.R., Thomer, M., Murnen, K.F., and Calvi, B.R. 2005. Levels of the origin-binding protein Double parked and its inhibitor Geminin increase in response to replication stress. J. Cell Sci. 118: 4207-4217.

McGarry, T.J. and Kirschner, M.W. 1998. Geminin, an inhibitor of DNA replication, is degraded during mitosis. Cell 93: 1043-1053.

McGuire, S.E., Mao, Z., and Davis, R.L. 2004. Spatiotemporal gene expression targeting with the TARGET and geneswitch systems in Drosophila. Sci. STKE 2004: pl6. doi: 10.1125/stke.2202004p16

Narbonne-Reveau, K., Senger, S., Pal, M., Herr, A., Richardson, H.E., Asano, M., Deak, P., and Lilly, M.A. 2008. APC/CFzr/ Cdh1 promotes cell cycle progression during the Drosophila endocycle. Development 135: 1451-1461.

Neufeld, T.P., de la Cruz, A.F., Johnston, L.A., and Edgar, B.A. 1998. Coordination of growth and cell division in the Drosophila wing. Cell 93: 1183-1193.

Pal, M., Nagy, O., Menesi, D., Udvardy, A., and Deak, P. 2007. Structurally related TPR subunits contribute differently to the function of the anaphase-promoting complex in Drosophila melanogaster. J. Cell Sci. 120: 3238-3248.

Parisi, T., Beck, A.R., Rougier, N., McNeil, T., Lucian, L., Werb, Z., and Amati, B. 2003. Cyclins E1 and E2 are required for endoreplication in placental trophoblast giant cells. EMBO $J$. 22: 4794-4803. 
Peters, J.M. 2006. The anaphase promoting complex/cyclosome: A machine designed to destroy. Nat. Rev. Mol. Cell Biol. 7: 644-656.

Pierce, S.B., Yost, C., Britton, J.S., Loo, L.W., Flynn, E.M., Edgar, B.A., and Eisenman, R.N. 2004. dMyc is required for larval growth and endoreplication in Drosophila. Development 131: 2317-2327.

Pines, J. 2006. Mitosis: A matter of getting rid of the right protein at the right time. Trends Cell Biol. 16: 55-63.

Quinn, L.M., Herr, A., McGarry, T.J., and Richardson, H. 2001. The Drosophila Geminin homolog: Roles for Geminin in limiting DNA replication, in anaphase and in neurogenesis. Genes \& Dev. 15: 2741-2754.

Reber, A., Lehner, C.F., and Jacobs, H.W. 2006. Terminal mitoses require negative regulation of $\mathrm{Fzr} / \mathrm{Cdh} 1$ by Cyclin A, preventing premature degradation of mitotic cyclins and String/Cdc25. Development 133: 3201-3211.

Reed, B.H. and Orr-Weaver, T.L. 1997. The Drosophila gene morula inhibits mitotic functions in the endo cell cycle and the mitotic cell cycle. Development 124: 3543-3553.

Reis, T. and Edgar, B.A. 2004. Negative regulation of dE2F1 by cyclin-dependent kinases controls cell cycle timing. Cell 117: 253-264.

Royzman, I., Austin, R.J., Bosco, G., Bell, S.P., and Orr-Weaver, T.L. 1999. ORC localization in Drosophila follicle cells and the effects of mutations in $\mathrm{dE} 2 \mathrm{~F}$ and dDP. Genes \& Dev. 13: 827-840.

Sauer, K., Knoblich, J.A., Richardson, H., and Lehner, C.F. 1995. Distinct modes of cyclin E/cdc2c kinase regulation and Sphase control in mitotic and endoreduplication cycles of Drosophila embryogenesis. Genes \& Dev. 9: 1327-1339.

Schaeffer, V., Althauser, C., Shcherbata, H.R., Deng, W.M., and Ruohola-Baker, H. 2004. Notch-dependent Fizzy-related/ $\mathrm{Hec} 1 / \mathrm{Cdh} 1$ expression is required for the mitotic-to-endocycle transition in Drosophila follicle cells. Curr. Biol. 14: 630-636.

Shibutani, S., Swanhart, L.M., and Duronio, R.J. 2007. Rbf1independent termination of E2f1-target gene expression during early Drosophila embryogenesis. Development 134: 467478.

Sigrist, S.J. and Lehner, C.F. 1997. Drosophila fizzy-related down-regulates mitotic cyclins and is required for cell proliferation arrest and entry into endocycles. Cell 90: 671-681.

Smith, A.V. and Orr-Weaver, T.L. 1991. The regulation of the cell cycle during Drosophila embryogenesis: The transition to polyteny. Development 112: 997-1008.

Tada, S., Li, A., Maiorano, D., Mechali, M., and Blow, J.J. 2001. Repression of origin assembly in metaphase depends on inhibition of RLF-B/Cdt1 by geminin. Nat. Cell Biol. 3: 107113.

Tanimoto, H., Itoh, S., ten Dijke, P., and Tabata, T. 2000. Hedgehog creates a gradient of DPP activity in Drosophila wing imaginal discs. Mol. Cell 5: 59-71.

Thacker, S.A., Bonnette, P.C., and Duronio, R.J. 2003. The contribution of E2F-regulated transcription to Drosophila PCNA gene function. Curr. Biol. 13: 53-58.

Weiss, A., Herzig, A., Jacobs, H., and Lehner, C.F. 1998. Continuous Cyclin E expression inhibits progression through endoreduplication cycles in Drosophila. Curr. Biol. 8: 239-242.

Weng, L., Zhu, C., Xu, J., and Du, W. 2003. Critical role of active repression by $\mathrm{E} 2 \mathrm{~F}$ and $\mathrm{Rb}$ proteins in endoreplication during Drosophila development. EMBO J. 22: 3865-3875.

White, A.E., Leslie, M.E., Calvi, B.R., Marzluff, W.F., and Duronio, R.J. 2007. Developmental and cell cycle regulation of the Drosophila histone locus body. Mol. Biol. Cell 18: 24912502.
Whittaker, A.J., Royzman, I., and Orr-Weaver, T.L. 2000. Drosophila double parked: A conserved, essential replication protein that colocalizes with the origin recognition complex and links DNA replication with mitosis and the down-regulation of S phase transcripts. Genes \& Dev. 14: 1765-1776.

Wohlschlegel, J.A., Dwyer, B.T., Dhar, S.K., Cvetic, C., Walter, J.C., and Dutta, A. 2000. Inhibition of eukaryotic DNA replication by geminin binding to Cdt1. Science 290: 23092312.

Wu, S., Huang, J., Dong, J., and Pan, D. 2003. hippo encodes a Ste-20 family protein kinase that restricts cell proliferation and promotes apoptosis in conjunction with salvador and warts. Cell 114: 445-456. 


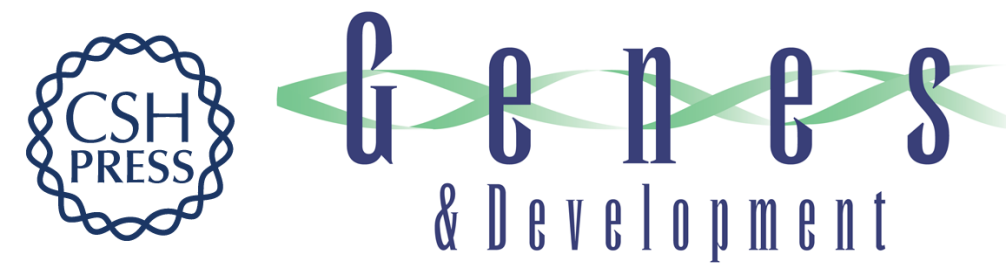

\section{The anaphase-promoting complex/cyclosome (APC/C) is required for rereplication control in endoreplication cycles}

Norman Zielke, Silvia Querings, Carmen Rottig, et al.

Genes Dev. 2008, 22:

Access the most recent version at doi:10.1101/gad.469108

Supplemental http://genesdev.cshlp.org/content/suppl/2008/06/11/22.12.1690.DC1
Material

References This article cites 65 articles, 35 of which can be accessed free at:

http://genesdev.cshlp.org/content/22/12/1690.full.html\#ref-list-1

License

Email Alerting

Receive free email alerts when new articles cite this article - sign up in the box at the top

Service

right corner of the article or click here.

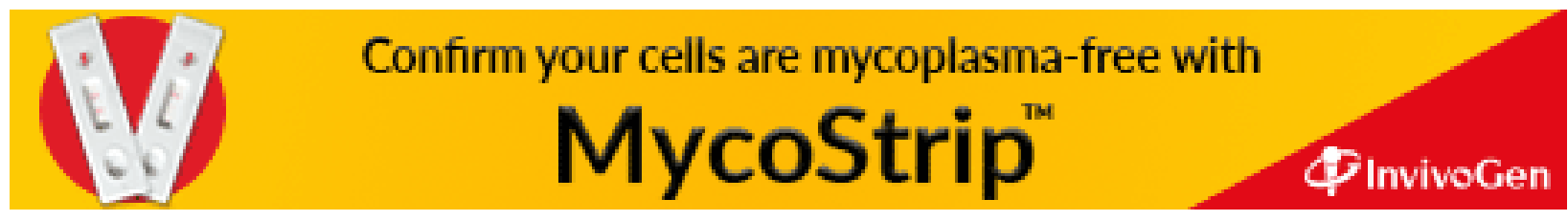

